\title{
Information Society in Extremadura
}

Towards a Future of Solidarity and More Freedom

Jose A. Diaz

Digital Literacy Plan, Junta of Extremadura

\begin{abstract}
This paper describes a strategic project for an information society in the Spanish Region of Extremadura. One important aspect of this project was the development of GNU/LinEx and associated Free Software. The paper describes the advantages of this Free Software.
\end{abstract}

Keywords: Public intranet, regions, educational technology network, Free Software.

\section{INTRODUCTION}

A strategic project in Extremadura to access the Information Society is based upon the principles of networking and technological literacy, and its main aim is better life quality for Extremadura inhabitants, from an equality and freedom viewpoint.

For this reason a series of actions have been taken in the Region. On the one hand a powerful communication infrastructure has been developed: the Regional Intranet. This intranet is able to connect more than 1400 points distributed throughout the 383 localities belonging to our Autonomous Community. On the other hand programs reaching educational and socioeconomic targets have been started.

The keystone of this strategy was clearly found in education. Information and communication technologies will contribute in a decisive way to improve the quality of education. So, the design of the Educational Technological Network (RTE) started off by providing one computer per two students in Secondary Schools. The Technological Literacy Plan (PAT) was also designed to take into account the needs of groups to whom this new 
technological world was less familiar. With this aim its 33 New Centres of Knowledge are distributed throughout the territory of Extremadura, especially in rural areas. Finally, in order to include the other social layers, Vivernet's task is that of supporting business in the digital era, the New Initiatives Promotion Centre being responsible for analysing and guiding the Information Society Strategies of Extremadura, depending on the present changing circumstances.

In this set of circumstances the GNU/LinEx Project (Free Software) is framed on this context as an answer to the need to avoid external dependencies, like proprietary software, which are not subject to any kind of public control.

\section{CONTEXT: THE SOCIO-ECONOMIC FEATURES OF EXTREMADURA}

Extremadura possesses a land area of $41,634 \mathrm{~km}^{2}$ and a population of $1,073,574$, meaning a population density of 25.78 inhabitants per $\mathrm{km}^{2}$. The regional territory makes up $8.3 \%$ of Spain but its population is $2.6 \%$ of the total. Population is distributed throughout 383 localities, but only Badajoz (136,316 inhabitants) has more than 100,000 inhabitants. $57 \%$ of people in Extremadura live in villages of less than 10,000 people. According to population, Cáceres is the second town with 82,034 inhabitants and the regional capital, Mérida is third with 51,056 inhabitants.

The economy of Extremadura has been undergoing an evolution in recent years. It was the best Spanish Autonomous Community in regard to European convergence in the areas of educational, social and business projects during the period 1985-1999. These projects are bringing about development, positioning the region at the same level of equality and freedom, based on the premise of introducing the region in the new technologies revolution. They are fostering the possibility of coping with the knowledge revolution now and in the future.

\section{THE INFORMATION SOCIETY GLOBAL STRATEGY OF EXTREMADURA}

In 1998 during the general politics debate the President of Extremadura, Juan Carlos Rodríguez Ibarra, announced that the Extremadura Government's efforts would be directed towards positioning the region among the leaders of the technological revolution known as the information society. Such a revolution would involve the whole population and it would 
be based upon the principles of networking and technological literacy, in order to improve citizens' welfare.

We had lost our chance to be included in the industrial revolutions and this was the moment to take advantage of an extraordinary chance to join this revolution. The knowledge society was not based on commodities like coal or oil, but information and computer networks. This situation positioned any individual person at the same level everywhere, and with the same successful opportunities, regardless of the place where the they lived.

The proposal was ambitious, and a series of actions was taken to provide the region with telecommunications infrastructures as well as with the necessary programs to reach the educational and socio-economic aims.

\subsection{Technological Framework: Intranet of Extremadura}

Development of the 'Intranet of Extremadura' started in 1999 with the main aim of providing high bandwidth access $(2 \mathrm{Mb})$ in regional localities through the public educational centres, public health centres and public administrative centres managed by the Government of Extremadura. At the end of 2001, the intranet was fully set through 1800 network points and all Extremadura localities, almost 400 , have access to high bandwidth and high speed Internet connections.

Technically speaking, the intranet is an optical fibre ring which connects each public administrative centre to a single access point which is located in the Data Processing Centre, Mérida. Radio link technology is used where there is a lack of optical fibre. In this way, there is a network of transmittingreceiving antennas throughout the region connecting the single access point to the different localities. In a similar way WIFI technology is used to connect separated buildings of each educational centre in certain localities where the intranet is accessed in a central building, but there are other dependent buildings around the same centre.

In the future there is a possibility of using the remaining bandwidth of the intranet to offer Internet connections to localities which lack high speed technologies like ADSL.

\subsection{Strategic Framework}

While the design of the regional intranet was being prepared, a set of programs were started off, addressed different areas of the Society of Extremadura. These programs cope with the technological literacy of the population, the promotion of business opportunities based on new technologies, the spreading of information society and new technologies, and improvement of the education system. 


\subsubsection{Technological Literacy Plan}

The Technological Literacy Plan (PAT) was first started by the Regional Government at the end of 1999. Its main aim was to provide each citizen with universal access to information technologies. The technological literacy process is based upon a structure of centres, called New Centres of Knowledge (NCC). Throughout the region there are 33 new centres of knowledge, especially in the most far-from-city rural areas and the outlying urban areas.

These centres offer to their users, mainly adults, training in new technologies according to their needs. Generally speaking they cope with basic use of computing tools. Remarkably, we could say that a high number of old people have learnt to use computers, to surf the web, and to manage their email accounts in these centres.

There is a computer technician, but also a public relations officer in each NCC. There are also seven computers with Internet connections and the usual peripherals - scanner, printer, digital camera, etc. Since GNU/LinEx was introduced the centres have used Free Software without having any problems. Since GNU/LinEx began 50,000 people have participated in events designed by the NCCs. The users have welcomed the operating system in a positive way.

\subsubsection{Vivernet}

Vivernet, "The New Era Business Laboratory", was born in the second half of 2000. Its main aim consists of promoting new technology entrepreneurs. In its headquarters, physically settled in Badajoz and Cáceres, the new business stays for an average of 15 months. The entrepreneurs receive legal and financial training, and also technological training.

Vivernet spreads the use of GNU/LinEx, and develops Free Software for the regional SMEs (Small and Medium Enterprises) in training courses and events related to this kind of business software.

\subsubsection{New Initiatives Promotion Centre}

Perhaps the New Initiatives Promotion Centre is a less known project because it works directly for the Public Service. It is responsible for analysing and defining Information Society strategies. It also promotes the use and development of information technology in different social areas. During the last 2 years the Centre has developed the eExtremadura program, which is an action financed by European funds. This program consists of joining together different regional agents - trade unions, enterprises, town 
councils, university - in order to develop new technology projects. In the second convocatory after the introduction of GNU/LinEx, more than 50 projects from a total of 400 mentioned use of GNU/LinEx or Free Software.

Today, the New Initiatives Promotion Centre is managing technical development of the GNU/LinEx project. This project has as partners the Education, Science and Technology Regional Ministry, the New Centres of Knowledge, Vivernet, and Fundecyt.

\subsubsection{Educational Technological Network}

The Educational Technological Network is the most characteristic project of the Education, Science and Technology Regional Ministry. Its main aim consists of introducing the educational system into the knowledge society. Action was taken to train teachers in the use of ICT, and to promote creation of content to be shared on the net (www.extremadurasi.org).

The most ambitious action consists of changing the concept of 'computing classroom' into one of 'computer into the classroom'. This means a ratio of one computer per two students in Secondary Education Centres, a fact that has an influence even in the architectural design of the new buildings. The classrooms are larger and the building structure supports the needs for electric and networking wiring. In the classroom, there is a new larger design of desk for computers and for the students' usual needs. In the old centres the design of the desk is slightly smaller.

Getting to this point, there was a need to solve the problem of software to be installed on the computers. Proprietary software was not a solution and an economical alternative for 80,000 machines was needed. That alternative was Free Software.

\section{GNU/LINEX PROJECT}

At the end of 2001 the intranet was fully operational, the projects of the Information Society Secretary were consolidated, and 14 new educational centres were almost finished, according to the 1-computer-per-2-student ratio. About 400 machines were to be introduced in the new centres.

We had not stopped growing when a big problem appeared. On the one hand as technological projects, there was an important software dependency, in most of cases owned by a single company. We had to spend a great sum to pay for the licences for each of the machines.

On the other hand, a more important aspect, the educational system had introduced and installed 80,000 machines which needed operating systems, but also productivity software to work with. 
The large amount we had to spend 'bit everybody's eye' and obviously the license costs were almost unreachable, particularly if we take periodical license updating of products into account. So, an alternative solution was needed. Instead of proprietary software the only possibility was Free Software.

We had being using Free Software already in server environments with excellent results. But we had not tested a Free Software solution for end users lacking computing knowledge. Our main need was a desktop solution based of Free Software and oriented to users with office automation requirements.

In this sense, about November 2001 we got in touch with the company Ándago. They had experience in implementation of Free Software in desktop machines in the Ministry of Public Service. Once the authorities of the Regional Ministry gave their approval, they started to develop the first version of GNU/LinEx.

\subsection{Be Legal, Copy LinEx}

This was the slogan for the GNU/LinEx launch on March $17^{\text {th }} 2002$. It makes reference to the advantages of using Free Software, and the possibility of redistributing the software and code with full freedom. LinEx first version was based on the Debian Potato distribution and included GNOME as a graphical environment. To be honest, the version had lots of limitations, but it was a starting point.

A couple of months later the first advantages of Free Software were proved. Two Secondary teachers had developed a new version of LinEx based on the first one developed by Ándago, with improvements in all aspects. These teachers were included in the GNU/LinEx technical staff, which depends on the Education, Science and Technology Regional Ministry. In September 2002 the GNU/LinEx 3.0 Woody version was presented in SIMO. It was based on Debian Woody and included GNOME 1.4 as a graphical interface.

\subsection{What is GNU/LinEx?}

Technically speaking, GNU/LinEx is a Free Software distribution based on Debian GNU/Linux. It includes a set of programs and a graphical interface, GNOME, oriented to non-technical end-users. Debian is a distribution created and developed around the world by a large group of voluntary programmers, researchers, and especially teachers. Debian was chosen because, on the one hand, there are not trademarks associated with 
this distribution and, on the other hand, because this initiative provides high quality standards to its software.

Nevertheless, the standard Debian distribution was not suitable for all our needs so the GNU/LinEx development team's work consisted of adapting Debian to the needs of the Government of Extremadura. The first task to be undertaken was selecting software to be included in the single CD-ROM that GNU/LinEx was distributed on. Out of almost 9000 software packages compounding Debian, 800 have been selected. These are the packages included in the GNU/LinEx Distribution. The next task was the development of a graphic installer to make the installing process as easy as possible to avoid inexperienced user's intervention. Finally, new icons have been added to the desktop. These icons make reference to the cultural background of Extremadura: for example, Espronceda is a word processor, Zurbarán is a graphic design tool, Guadalupe is an email program.

Once we did this we gained an easy-to-install product with a variety of productivity software able to cope with almost every need of an office or an educational system user.

Today, the main technical features of GNU/LinEx, version 3.0r1+g2.2, are as follows:

- Linux Kernel 2.4.20

- Graphical Interface GNOME 2.2

- A set of GNU free applications:

(Semi) automatic graphic installer

Word processors

Spreadsheet

Presentations

Accessories

Web browser

Email program

Instant messenger

Domestic financial management

Image editor

$\mathrm{VNC}$

and much more...

\subsection{GNU/LinEx on the Net: www.linex.org}

Running in parallel to GNU/LinEx presentation was the www.linex.org portal. Information on the project is collected here and in this web site you can download GNU/LinEx from different servers, and obtain technical support for users' frequently asked questions. You can also access the GNU/LinEx documentation, manuals and tutorials. 
A Technical Support Service will be accessible through the web portal very soon. Users will be able to ask questions which would be answered through the web site, as well as via their emails.

\section{GNU/LINEX IS A COLLECTIVE PROJECT}

When the Regional Government started off the project of working with Free Software in the Public System, GNU/LinEx became vey well known on the net. From this time the Government decided to adopt Free Software in a decisive way. Certainly regarding the educational environment, Extremadura is a world pioneer in installing a free operating system in classrooms. The GNU/LinEx project has appeared on the front page of national and international newspapers such as the Washington Post or the International Herald Tribune. It is of interest to several Governments and to national and international organizations that have declared their intention of cooperating with the development of this initiative.

The first collaboration for the development of Free Software was a Protocol signed by the Regional Governments of Extremadura and Andalusia in March 2003. According to the Protocol, Andalusia would use Extremadura's advances to develop their own distribution, called GNU/Guadalinex, to be launched in September 2003. To produce the protocol, Andalusia and Extremadura have joined a shared technical team to coordinate and share advances in both Autonomous Communities.

Beyond the Spanish frontiers, there are lots of cooperative agreements with the Government of Brasil, as well as contacts with Argentina, Colombia and Peru.

The GNU/LinEx project is not only supported by Governments, but also by user and business associations: GULEX or SINUH, Extremadura GNU/LinEx user associations, or Hispalinux, the Spanish user association.

The business role in this project is a most relevant one. From the very beginning, we observed the use of Free Software in a local enterprise environment as one of the main advantages, especially for the already limited technological sector. In this respect one of the results is development of tools oriented to SME financial management, accounting, payslips, etc.

Other companies, such as OKI, contacted the GNU/LinEx project. They have contributed by the development of their printer software installers for GNU/LinEx. ACER has included GNU/LinEx as pre-installed software in their laptops. Lambdaux Software contributes interchanging technologies between GNU/LinEx and Lux, the pre-installed distribution in AIRIS laptop models. Summing up, big companies pay attention to the important base of users of Free Software as a business opportunity. 


\section{FREE SOFTWARE FOR THE PUBLIC SERVICE}

In Extremadura, our experience in using and distributing GNU/LinEx brings us to several conclusions about the introduction of Free Software in the Public Service, and there are more pros than cons:

(a) Public funds management. The Public Services has a duty to manage the citizens' money to obtain the maximum benefit for everybody. Investing in Free Software, the Public Service System gives society back the profits in font code appearance. This is a way to invest in local R\&D because the Public Service is using the services of software companies in the region. As the font code is open the application costs are more economical.

(b) GPL vs. Proprietary License. When buying proprietary software licenses, the Public Service System depends on the business policies of a single supplier. These could change at any moment. But GPL licenses make independence from suppliers possible, so that the Public Service could negotiate and make a choice of the best service.

(c) Third party software licenses are not compulsory. If the Public Service System uses proprietary software to communicate with citizens, citizens are obliged to use proprietary software to communicate with the Public Service. But if Free Software is used the citizens have an option to chose.

(d) The Public Service has also a responsibility as a big consumer. If it takes action it could support technological sectors based on Free Software.

(e) Summing up, Free Software promotes positive attitudes like knowledge sharing and network cooperation. It is a new, profitable business model based on services related to software. This gives regions like Extremadura the opportunity to be at the same level as other regions in the world.

In light of these conclusions, the question to be answered is: Why do we have to rent proprietary software if we have a choice for Free Software? 